\title{
Clinical diagnostic criteria of autoimmune pancreatitis: revised proposal
}

\author{
Kazuichi Okazaki ${ }^{1}$, Shigeyuki Kawa ${ }^{2}$, Terumi Kamisawa ${ }^{3}$, Satoru Naruse ${ }^{4}$, Shigeki Tanaka ${ }^{5}$, Isao Nishimori ${ }^{6}$, \\ Hirotaka Ohara ${ }^{7}$, Tetsuhide Ito ${ }^{8}$, Seiki Kiriyama ${ }^{9}$, Kazuro Inui ${ }^{10}$, Tooru Shimosegawa ${ }^{11}$, Masaru Koizumi ${ }^{12}$, \\ Koichi Suda ${ }^{13}$, Keiko Shiratori ${ }^{14}$, Koji Yamaguchi ${ }^{15}$, Taketo Yamaguchi ${ }^{16}$, Masanori SugiYama ${ }^{17}$, \\ and Макото ОтsUкI ${ }^{18}$ \\ ${ }^{1}$ The Third Department of Internal Medicine, Kansai Medical University, Moriguchi, Japan \\ ${ }^{2}$ Center for Health, Safety and Environmental Management, Shinshu University, Matsumoto, Japan \\ ${ }^{3}$ Internal Medicine, Tokyo Metropolitan Komagome Hospital, Tokyo, Japan \\ ${ }^{4}$ Department of Gastroenterology, Nagoya University Graduate School of Medicine, Nagoya, Japan \\ ${ }^{5}$ Second Department of Internal Medicine, Showa University School of Medicine, Tokyo, Japan \\ ${ }^{6}$ Department of Gastroenterology and Hepatology, Kochi, Medical School, Nankoku, Japan \\ ${ }^{7}$ Department of Internal Medicine and Bioregulation, Nagoya City University Graduate School of Medical Sciences, Nagoya, Japan \\ ${ }^{8}$ Department of Medicine and Bioregulatory Science, Graduate School of Medical Sciences, Kyushu University, Fukuoka, Japan \\ ${ }^{9}$ Department of Gastroenterology, Ogaki Municipal Hospital, Ogaki, Japan \\ ${ }^{10}$ Department of Internal Medicine, Fujita Health University, Second Teaching Hospital, Nagoya, Japan \\ ${ }^{11}$ Division of Gastroenterology, Tohoku University Graduate School of Medicine, Sendai, Japan \\ ${ }^{12}$ Kurihara Central Hospital, Kurihard, Japan \\ ${ }^{13}$ Department of Pathology, Juntendo University School of Medicine, Tokyo, Japan \\ ${ }^{14}$ Department of Gastroenterology, Tokyo Women's Medical University School of Medicine, Tokyo, Japan \\ ${ }^{15}$ Department of Surgery and Oncology, Graduate School of Medical Sciences, Kyushu University, Fukuoka, Japan \\ ${ }^{16}$ Department of Medicine and Clinical Oncology, Graduate School of Medicine, Chiba University, Chiba, Japan \\ ${ }^{17}$ Department of Surgery, Kyorin University School of Medicine, Tokyo, Japan \\ ${ }^{18}$ Third Department of Internal Medicine, University of Occupational and Environmental Health, Japan School of Medicine, Kokura, Japan
}

Members of the Autoimmune Pancreatitis Diagnostic Criteria Committee, the Research Committee of Intractable Diseases of the Pancreas, supported by the Japanese Ministry of Health, Labour and Welfare (Chairman, Makoto Otsuki):

K. Okazaki, S. Kawa, T. Kamisawa, S. Naruse, S. Tanaka, I. Nishimori, H. Ohara, T. Ito, S. Kiriyama, K. Inui, T. Shimosegawa, M. Koizumi, K. Suda, and M. Otsuki

Members of the Autoimmune Pancreatitis Diagnostic Criteria Committee, the Japan Pancreas Society:

K. Shiratori, M. Sugiyama, K. Yamaguchi, and T. Yamaguchi

Key words: autoimmune pancreatitis, diagnostic criteria, lymphoplasmacytic sclerosing cholangitis, IgG4

\section{Introduction}

In 1961, Sarles et al. ${ }^{1}$ asked the following question regarding the particular cases of pancreatitis with hypergammaglobulinemia: "Chronic inflammatory sclerosis of the pancreas-an autoimmune pancreatic disease?" As similar cases were rarely observed, a relationship between such pancreatitis and autoimmunity was viewed skeptically during the following several decades. In 1992, Toki et al. ${ }^{2}$ have reported 4 cases with unusual diffuse irregular narrowing of the main pancre-

Received: June 26, 2006 / Accepted: June 27, 2006

Reprint requests to: K. Okazaki, The Third Department of Internal Medicine, Division of Gastroenterology and Hepatology, Kansai Medical University, 2-3-1 Shinmachi, Hirakata 573-1191, Japan atic duct and diffuse enlargement of the entire pancreas due to lymphocyte infiltration. In 1995, Japanese investigators $^{3}$ firstly proposed a concept of "autoimmune pancreatitis (AIP)", in which the patients showed diffusely enlarged pancreas, narrowing pancreatogram, increased serum IgG, presence of autoantibodies, fibrotic changes with lymphocytic infiltration and steroidal efficacy. Thereafter, many AIP cases have been reported from Japan, and AIP has been accepted as a new clinical entity. ${ }^{4,5}$ The histopathological findings of AIP show massive infiltration of lymphoplasmacytes with fibrosis, which is consistent with lymphoplasmacytic sclerosing pancreatitis (LPSP). ${ }^{6}$ Many Japanese investigators have paid great attention to AIP, especially with regard to its unique pancreatic images, ${ }^{2} \mathrm{IgG} 4,{ }^{7}$ diseaseassociated autoantibodies, ${ }^{8}$ extrapancreatic lesions, ${ }^{6,9-14}$ and steroidal efficacy. ${ }^{14,15}$

Currently in Japan, diagnosis of AIP is based on the "diagnostic criteria 2002 of autoimmune pancreatitis"16 proposed by the Japan Pancreas Society. However, the accumulation of many AIP cases shows that the 
concept of AIP has changed slightly to include extrapancreatic lesions and associated disorders, which suggests that the current diagnostic criteria are becoming inadequate.

In 2003, the Research Committee of Intractable Diseases of the Pancreas, supported by the Japanese Ministry of Health, Labour and Welfare (Chairman, M. Otsuki), began to review the current diagnostic criteria in light of recently acquired information and knowledge. The team organized a working group (WG), consisting of the team members and researchers specializing in autoimmune pancreatitis, to develop a proposal for the revision of the current diagnostic criteria. On 7 October 2005 and 22 April 2006, the Research Committee of Intractable Diseases of the Pancreas and the Japan Pancreas Society jointly held open forums to discuss the proposed amendments.

This report describes the background of the proposed amendments and the final proposal for the revised version of the clinical diagnostic criteria of AIP.

\section{Background of the amendment of the diagnostic criteria proposed by the working group of the Research Committee of Intractable Diseases of the Pancreas}

Table 1 shows the diagnostic criteria 2002 of AIP proposed by the Japan Pancreas Society. ${ }^{16}$ The members of the WG accumulated 147 cases of AIP and identified the cases that did not fulfill the current diagnostic criteria but were strongly suspected to be AIP. It then evaluated those cases in detail using imaging, laboratory, and pathological findings, and unanimously rediagnosed some of them as AIP. Using the analyzed data, the members then summarized the current status and problems of AIP in Japan, as outlined below. After reviewing the material in an expanded open discussion in October 2004 in Fukuoka City, the Research Committee of Intractable Diseases of the Pancreas amended the current diagnostic criteria for AIP. Major discussions in this open meeting are summarized below.

Table 1. Diagnostic criteria 2002 of autoimmune pancreatitis by the Japan Pancreas Society

1. Diffuse or segmental narrowing of the main pancreatic duct with irregular wall (more than $1 / 3$ length of the entire pancreas) and diffuse or localized enlargement of the pancreas by imaging studies

2. High serum $\gamma$-globulin and/or IgG, or the presence of autoantibodies, such as antinuclear antibodies and rheumatoid factor

3. Marked interlobular fibrosis and prominent infiltration of lymphocytes and plasma cells in the periductal area, occasionally with lymphoid follicles, in the pancreas

For diagnosis, criterion 1 must be present together with criterion 2 and/or 3

Table 2. Clinicopathological features of autoimmune pancreatitis

Age and sex

Clinical symptoms

Laboratory data

Imaging of the pancreaticobiliary system

Histopathologic findings of the pancreas

Occasional extrapancreatic lesions

Occasional association with other autoimmune diseases

Effective steroid therapy

Prognosis
More common in elderly males

Mild abdominal symptoms, usually without acute attacks of pancreatitis Occasional existence of obstructive jaundice

Increased levels of serum $\gamma$-globulin, IgG or IgG4

Presence of autoantibodies

Increased hepatobiliary and/or pancreatic enzymes Impaired exocrine and endocrine pancreatic function

Enlargement of the pancreas

Irregular narrowing of the pancreatic duct

Stenosis of intrapancreatic bile duct

Sclerosing cholangitis similar to PSC

Interlobular fibrosis, occasionally intralobular fibrosis

Atrophy of acini

Infiltration of lymphocyte and IgG4-positive plasma cells

Obliterative phlebitis

Sclerosing cholangitis similar to PSC

Sclerosing sialadenitis

Retroperitoneal fibrosis

Interstitial nephritis

Chronic thyroiditis

Interstitial pneumonia

Lymphadenopathy (mediastinum/peritoneal cavity)

Unclear long-term prognosis

Pancreatic stone formation in some cases 


\section{Concept and definition of AIP}

1. The following extrapancreatic lesions may be associated with AIP: biliary lesions, sialadenitis, retroperitoneal fibrosis, enlarged celiac and hilar lymph nodes, chronic thyroiditis, and interstitial nephritis $^{9-14}$ (Table 2). AIP may be a systemic disorder.

2. Sclerosing cholangitis associated with AIP is different from primary sclerosing cholangitis (PSC), because of its effective response to steroid therapy and of the presence of IgG4-positive plasma cell infiltration. ${ }^{9-14}$

3. Sialadenitis coexisting with AIP is negative for both anti-SSA and anti-SSB antibodies and shows IgG4positive plasma cell infiltration, suggesting that it is different from typical Sjögren's syndrome but is similar to the sclerosing sialadenitis observed in Mikulicz's disease and Küttner's tumor., ${ }^{9,10,14}$

4. In Western countries, ulcerative colitis and the formation of tumors associated with pancreatic lesions are more frequently observed than in Japan. ${ }^{4}$ Moreover, pancreatic duct lesions with infiltration of leukocytes as well as LPSP are sometimes observed in Western countries ${ }^{17,18}$ but not in Japan. These findings may thus be somewhat contrary to the definition and concept of the disease adopted in Japan.

5. The biggest problem in diagnosing AIP is how to distinguish it from pancreatic or biliary cancer. With the current diagnostic criteria for AIP, many cases that seem to be AIP are excluded, as the criteria were established to rule out false positive cases but to confidently diagnose definite cases of AIP.,14

\section{Image findings}

1. Some cases are consistent with the hematological and histopathological findings of AIP, even though the pancreatic image data show that the lesions are localized to less than one-third of the pancreas. ${ }^{16}$

2. Some cases fulfill only the image diagnostic criteria for AIP but respond to steroid therapy. ${ }^{14}$

\section{Laboratory findings}

1. Patients with AIP frequently show an increased level of serum $\operatorname{IgG} 4{ }^{7}$ a subclass of $\operatorname{IgG}$, although the pathophysiological significance is still unclear. Serum levels of IgG4 in 147 patients with AIP (602.8 \pm $609.1 \mathrm{mg} / \mathrm{dl}$ ) were significantly higher than those in other, control patients $(52.4 \pm 57.2 \mathrm{mg} / \mathrm{dl})$ (Fig. 1).

2. Using receiver operating characteristic curves for the 147 cases of AIP and 180 cases of other diseases, the cutoff value for the serum $\mathrm{IgG} 4$ was determined to be $128 \mathrm{mg} / \mathrm{dl}$ (Fig. 2), which confirmed the validity of the evidenced value of $134 \mathrm{mg} / \mathrm{dl}^{7}$

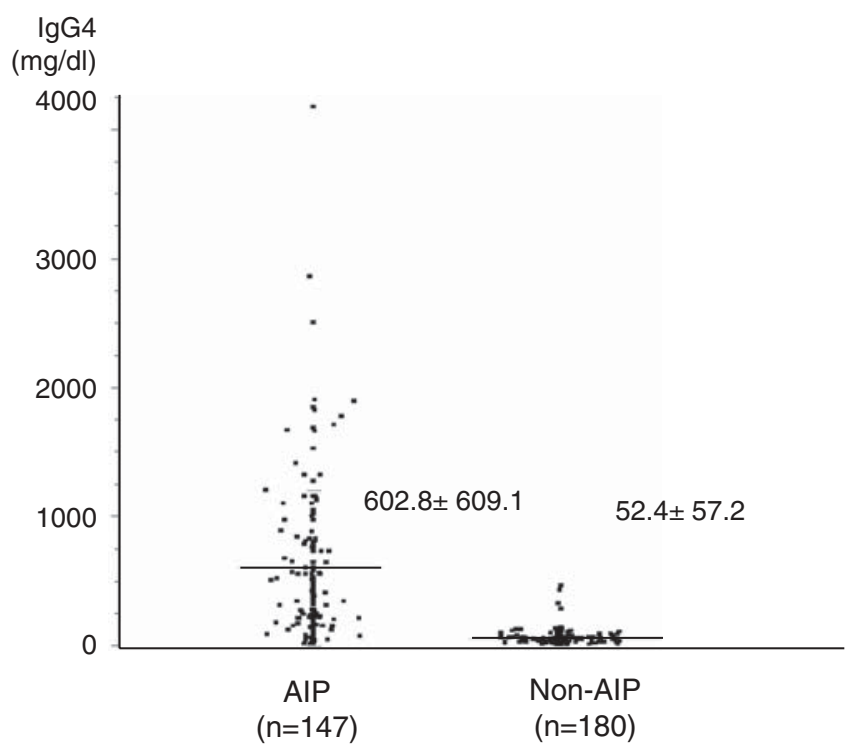

Fig. 1. Serum levels of $\mathrm{IgG} 4$ in 147 patients with autoimmune pancreatitis $(A I P)(602.8 \pm 609.1 \mathrm{mg} / \mathrm{dl})$ were significantly higher than those $(52.4 \pm 57.2 \mathrm{mg} / \mathrm{dl})$ in 180 control patients (103 cases of alcoholic chronic pancreatitis, 18 of pancreatic cancer, 13 of primary sclerosing cholangitis, 11 of idiopathic chronic pancreatitis, 5 of cholangiocarcinoma, 2 of Sjögren's syndrome, and 28 other)

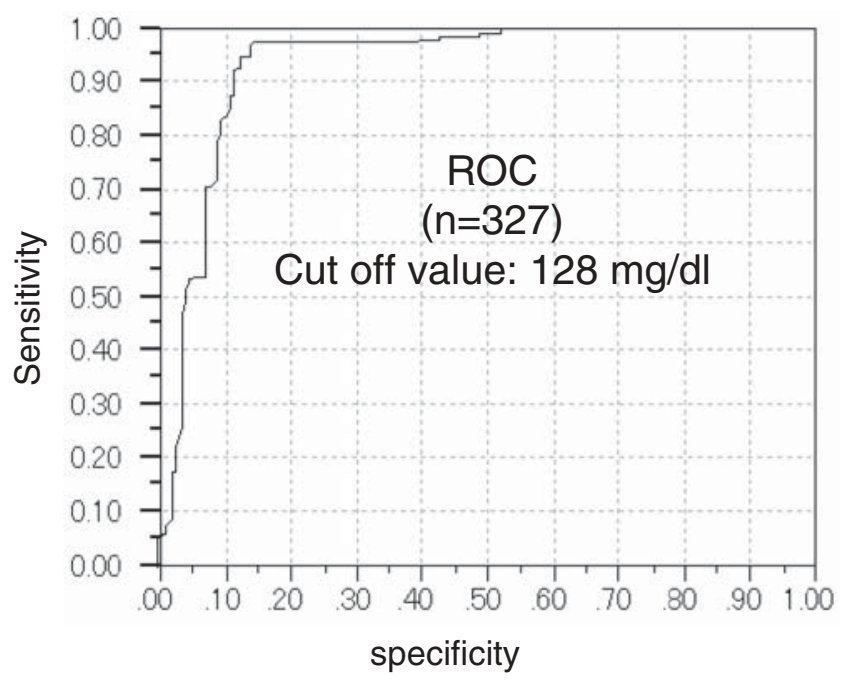

Fig. 2. The receiver operating characteristic $(R O C)$ curves. The ROC curves for the 147 cases of AIP and 180 cases of control diseases, the cutoff value for the serum $\mathrm{IgG} 4$ was determined to be $128 \mathrm{mg} / \mathrm{dl}$

\section{Pathological findings}

1. There is growing evidence that patients with AIP exhibit histopathological symptoms of lymphoplasmacytic sclerosing pancreatitis., ${ }^{4,6,17,18}$ That is, AIP patients show infiltration of lymphocytes and plasma 
cells, and obliterative phlebitis. In lymphocyte infiltration, $\mathrm{T}$ cells are often predominant, and plasma cells are IgG4-positive. ${ }^{14}$

2. European and U.S. reports of the destruction of the pancreatic duct epithelium in the presence of predominant neutrophils (idiopathic ductcentric chronic pancreatitis ${ }^{17}$ or granulocyte epithelial lesion ${ }^{18}$ ) have not been confirmed in Japan owing to insufficient study and will be a topic of further study.

3. Pathologically, extrapancreatic lesions are similar to the fibrosis and the lymphoplasmacytic infiltration seen in the pancreatic lesions.

\section{Prognosis}

1. Some patients with AIP form pancreatic stones, and the long-term prognosis of AIP is still unclear. ${ }^{14}$

\section{Final revision based on the discussion in the open forums}

In two recently held open forums, the committee members vigorously exchanged opinions about the amendments proposed by the Research Committee of Intractable Diseases of the Pancreas, and unanimously agreed to the finalized amendments (Table 3). The medical treatment guidelines in the current diagnostic criteria have been deleted from the proposed amendments. Those issues that were discussed in the open forums but not included in the proposed amendments are noted below as topics for future consideration.

1. Cases of AIP reported from Western countries are more often associated with ulcerative colitis and the formation of tumors compared with Japanese cases; these associations are somewhat contrary to the definition and concept of the disease adopted in

Table 3. Clinical Diagnostic Criteria of Autoimmune Pancreatitis (revised proposal) (Proposed by the Research Committee of Intractable Diseases of the Pancreas, supported by the Japanese Ministry of Health, Labour and Welfare, and the Japan Pancreas Society)

It is suspected that the pathogenesis of autoimmune pancreatitis (AIP) involves autoimmune mechanisms. Currently, the main characteristic findings of observed cases of AIP are the diffuse enlargement of the pancreas and the narrowing of the pancreatic duct, associated with findings suggestive of the involvement of autoimmune mechanisms such as increased levels of $\gamma$-globulin and $\mathrm{IgG}$, the presence of autoantibodies, and an effective response to steroid therapy. In some cases, AIP shows extrapancreatic manifestations such as sclerosing cholangitis, sclerosing sialadenitis, and retroperitoneal fibrosis, suggesting that AIP is a systemic disease. In Western countries, AIP is more frequently observed in association with ulcerative colitis and tumor formation, which observations are somewhat contrary to the definition and concept of the disease adopted in Japan.

Patients with AIP often experience discomfort in the epigastrium, obstructive jaundice due to bile duct stricture, and diabetes mellitus. AIP is more common in middle-aged and elderly men. Although long-term prognosis of the disease is not clear, pancreatic stone formation has been found in some cases.

When diagnosing AIP, it is important to differentiate it from neoplastic lesions such as pancreatic or biliary cancers, and to avoid facile therapeutic diagnosis by steroid administration. The present criteria, therefore, are based on the minimum consensus features of AIP in order to avoid the misdiagnosis of pancreatic or biliary cancer as far as possible but not to pick up suspicious cases of AIP.

I. Clinical diagnostic criteria

1. Diffuse or segmental narrowing of the main pancreatic duct with irregular wall and diffuse or localized enlargement of the pancreas by imaging studies, such as abdominal ultrasonography (US), computed tomography (CT), and magnetic resonance imaging (MRI).

2. High serum $\gamma$-globulin, IgG, or IgG4, or the presence of autoantibodies, such as antinuclear antibodies and rheumatoid factor.

3. Marked interlobular fibrosis and prominent infiltration of lymphocytes and plasma cells in the periductal area, occasionally with lymphoid follicles in the pancreas.

Diagnosis of autoimmune pancreatitis is established when criterion 1, together with criterion 2 and/or 3 , are fulfilled.

However, it is necessary to exclude malignant diseases such as pancreatic or biliary cancers.

Description notes

A. Imaging studies

1. Diffuse or localized swelling of the pancreas

Abdominal US, CT, and/or MRI show diffuse or localized swelling of the pancreas.

a. On US, pancreatic swelling is usually hypoechoic, sometimes with scattered echogenic spots.

b. Contrast-enhanced CT generally shows delayed enhancement similar to a normal pancreas with sausage-like enlargement, and/or a capsular-like low-density rim.

c. MRI shows diffuse or localized enlargement of the pancreas with lower density in the T1-weighed image and higher density in the T2-weighed image compared with the corresponding liver image. 
Table 3. Continued

2. Narrowing of the pancreatic duct

The main pancreatic duct shows diffuse or localized narrowing.

a. Unlike obstruction or stricture, narrowing of the pancreatic duct extends over a larger range, where the duct is narrowed with irregular walls. In typical cases, more than one-third of the entire length of the pancreatic duct is narrowed. Even in cases where the narrowing is segmental and extends to less than one-third of the total length, the upper part of the main pancreatic duct rarely shows notable dilatation.

b. When the pancreatic images show typical findings but laboratory data do not, AIP is possible. However, without histopathological examination, it is difficult to distinguish AIP from pancreatic cancer.

c. To obtain images of the pancreatic duct, it is necessary to use endoscopic retrograde cholangiopancreatography in addition to direct images taken during an operation or of specimens. Currently, it is difficult to depend for the diagnosis on magnetic resonance cholangiopancreatography.

3. The pancreatic image findings described above may be observed retrospectively from the time of diagnosis.

B. Laboratory data

1. In many cases, patients with AIP show increased levels of serum $\gamma$-globulin, IgG, or IgG4. High serum IgG4, however, is not specific to AIP, since it is also observed in other disorders such as atopic dermatitis, pemphigus, or asthma. Currently, the significance of high serum IgG4 in the pathogenesis and the pathophysiology of AIP is unclear.

2. Although increased levels of serum $\gamma$-globulin $(\geq 2.0 \mathrm{~g} / \mathrm{dl}), \operatorname{IgG}(\geq 1800 \mathrm{mg} / \mathrm{dl})$, and IgG4 ( $\geq 135 \mathrm{mg} / \mathrm{dl}) \mathrm{may}$ be used as a criterion for the diagnosis of AIP, further studies are necessary.

3. Autoantibodies such as antinuclear, anti-lactoferrin, anti-carbonic anhydrase antibody and rheumatoid factor are often detected in patients with AIP.

C. Pathohistological findings of the pancreas

1. Fibrotic changes associated with prominent infiltration of lymphocytes and plasma cells, occasionally with lymphoid follicles, are observed. In many cases, infiltration of IgG4-positive plasma cells is observed.

2. Lymphocytic infiltration is prominent in the periductal area, together with interlobular fibrosis, occasionally including intralobular fibrosis.

3. Inflammatory cell infiltration involves the ducts and results in diffuse narrowing of the pancreatic duct with atrophy of acini.

4. Obliterative phlebitis is often observed.

5. Although fine-needle biopsy under ultrasonic endoscopy is useful for differentiating AIP from malignant tumors, diagnosis may be difficult if the specimen is too small.

D. Endocrine and exocrine function of the pancreas

Some patients with AIP show a decline of exocrine pancreatic function and develop diabetes mellitus. In some cases, steroid therapy improves endocrine and exocrine pancreatic dysfunction.

II. Relationship to extrapancreatic lesions and other associated disorders

AIP may be associated with sclerosing cholangitis, sclerosing sialadenitis, or retroperitoneal fibrosis. Most AIP patients with sclerosing sialadenitis show negativity for both anti-SSA and anti-SSB antibodies, which may suggest that AIP differs from Sjögren's syndrome. Sclerosing cholangitis-like lesions accompanying AIP and primary sclerosing cholangitis respond differently to steroid therapy and have different prognoses, suggesting that they are not the same disorder. Further studies are necessary to clarify the role of autoimmune mechanisms in AIP.

Japan. ${ }^{4,9,17,18}$ These points were excluded from the Japanese notes, because in Japan, the basic concepts of AIP are generally accepted as pancreatitis featuring abnormal immunity, an enlarged pancreas, and a narrowing of the pancreatic duct. However, these controversial points were considered for inclusion in the introduction to the English notes because they were deemed necessary to clarify the inconsistencies between the findings of foreign and domestic researchers.

2. It was argued that AIP can be diagnosed by imaging data and the effective response to steroid therapy alone. This argument, however, was excluded because of two problems. First, no other autoimmune disorders use steroidal effect for diagnosis except for the scoring system for autoimmune hepatitis, in which the steroidal effect is not used for the differential diagnosis of malignancy. Second, above all, there was a concern that its adoption might encourage the use of facile therapeutic diagnostic techniques just to distinguish AIP from malignant tumors such as pancreatic cancer.

3. There was an argument as to whether the proposal should specify methods for ruling out other pancreatic disorders and pancreatic/biliary cancers. It was decided that each institute may decide the methods at their discretion, because standardization is rather difficult. 


\section{Conclusion}

The current concept of AIP including extrapancreatic lesions and associated disorders suggests that AIP may be a systemic disorder. As the concept of the disease has undergone changes, a revised version of the clinical diagnostic criteria of AIP in Japan has been proposed. Although it is not possible to establish diagnostic criteria that satisfy everyone, the Japanese criteria are based on the minimum consensus features of AIP in order to avoid the misdiagnosis of malignancy as far as possible, but not to pick up suspicious cases of AIP.

\section{References}

1. Sarles H, Sarles JC, Muratore R, Guien C. Chronic inflammatory sclerosis of the pancreas-an autoimmune pancreatic disease? Am J Dig Dis 1961;6:688-98.

2. Toki F, Kozu T, Oi I, Nakasako T, Suzuki M, Hanyu F, et al. An unusual type of chronic pancreatitis showing diffuse irregular narrowing of the entire main pancreatic duct on ERCP - a report of four cases. Endoscopy 1992;24:640.

3. Yoshida K, Toki F, Takeuchi T, Watanabe S, Shiratori K, Hayashi N. Chronic pancreatitis caused by autoimmune abnormality. Proposal of concept of autoimmune pancreatitis. Dig Dis Sci 1995;40: 1561-8.

4. Pearson RK, Longnecker DS, Chari ST, Smyrk TC, Okazaki K, Frulloni L, et al. Controversies in clinical pancreatology: autoimmune pancreatitis: does it exist? Pancreas 2003;27:1-13.

5. Okazaki K, Uchida K, Chiba T. Recent concept of autoimmunerelated pancreatitis J Gastroenterol 2001;36:293-302.

6. Kawaguchi K, Koike M, Tsuruta K, Okamoto A, Tabata I, Fujita W. Lymphoplasmacytic sclerosing pancreatitis with cholangitis: a variant primary sclerosing cholangitis extensively involving pancreas. Hum Pathol 1991;22:387-95.

7. Hamano H, Kawa S, Horiuchi A, Unno H, Furuya N, Akamatsu $\mathrm{T}$, et al. High serum IgG4 concentrations in patients with sclerosing pancreatitis. N Engl J Med 2001;344:732-8.
8. Okazaki K, Uchida K, Ohana M, Nakase H, Uose S, Inai M, et al. Autoimmune-related pancreatitis is associated with autoantibodies and a Th1/Th2 type cellular immune response. Gastroenterology 2000;118:573-81.

9. Kamisawa T, Okamoto A, Funata N. Clinicopathological features of autoimmune pancreatitis in relation to elevation of serum IgG4. Pancreas 2005;31:28-31.

10. Kamisawa T, Egawa N, Nakajima H, Tsuruta K, Okamoto A. Extrapancreatic lesions in autoimmune pancreatitis. J Clin Gastroenterol 2005;39:904-7.

11. Ohara H, Nakazawa T, Sano H, Ando T, Okamoto T, Takada H, et al. Systemic extrapancreatic lesions associated with autoimmune pancreatitis. Pancreas 2005;31:232-7.

12. Nakazawa T, Ohara H, Sano H, Ando T, Aoki S, Kobayashi S, et al. Clinical differences between primary sclerosing cholangitis and sclerosing cholangitis with autoimmune pancreatitis. Pancreas 2005;30:20-5.

13. Hamano H, Kawa S, Uehara T, Ochi Y, Takayama M, Komatsu $\mathrm{K}$, et al. Immunoglobulin G4-related lymphoplasmacytic sclerosing cholangitis that mimics infiltrating hilar cholangiocarcinoma: part of a spectrum of autoimmune pancreatitis? Gastrointest Endosc 2005;62:152-7.

14. Okazaki K, Uchida K, Matsushita M, Takaoka M. Autoimmune pancreatitis. Intern Med 2005;44:1215-23.

15. Nishimori I, Tamakoshi A, Kawa S, Tanaka S, Takeuchi K, Kamisawa T, et al. Influence of steroid therapy on the course of diabetes mellitus in patients with autoimmune pancreatitis: findings from a nationwide survey in Japan. Pancreas 2006;32:244-8.

16. Members of the Criteria Committee for Autoimmune Pancreatitis of the Japan Pancreas Society. Diagnostic criteria for autoimmune pancreatitis by the Japan Pancreas Society. J Jpn Pancreas (Suizou) 2002;17:587.

17. Notohara K, Burgart LJ, Yadav D, Chari S, Smyrk TC. Idiopathic chronic pancreatitis with periductal lymphoplasmacytic infiltration: clinicopathologic features of 35 cases. Am J Surg Pathol 2003;27:1119-27.

18. Zamboni G, Luttges J, Capelli P, Frulloni L, Cavallini G, Pederzoli $\mathrm{P}$, et al. Histopathological features of diagnostic and clinical relevance in autoimmune pancreatitis: a study on 53 resection specimens and 9 biopsy specimens. Virchows Arch 2004;445: $552-63$. 